\title{
Modelling Reliability Based Optimization Design for Water Distribution Networks
}

\author{
Mohamed Abdel Moneim \\ Holding Company for Water and Wastewater, \\ Arab Republic of Egypt
}

\section{Introduction}

There is a growing concern on the capacity of water utilities via governmental regulatory agencies regarding potential optimization and reliability for water distribution network. Generally, water distribution networks comprise about $60 \%$ of the total budget for a complete framework of a water supply system. According to this fact, achieving an optimum solution for water distribution networks as models' outcome of reliability-based optimization design has become the great concern to save considerable amount of allocated budget. During the last decade many authors were interested in studying optimization and reliability for water distribution networks that include solving non-linear hard problem of the network hydraulic equations. The optimization and reliability models of water distribution networks have number of varieties in studying aspects that include efficiency, accuracy, different sizes/scales of networks, and the consumed run time to define the optimum solution. During the current decade, considerable amount of attention has been given to reliability of water distribution networks in conjunction with the optimization to achieve maximum benefits with the minimum cost. This concern has been extended to cover the risk management for water distribution networks as a way to embark on facing the shortage of water resources all over the world or improving asset management programs. The main objective of this chapter is to develop standalone model divided into four submodels using MATLAB environment programming language. The developed model and its corresponding sub-models would acquaint an optimum solution for a given water distribution network that achieve both least cost design and reliability based optimization design in the mean time. The main model is called RELOPT and can be used as a tool to implement: modeling reliability-based optimization design, deterioration analysis of water pipe networks, risk analysis and assessment, and decision support system. RELOPT is integrated with four sub-models those are: optimization search engine model that is based on a new technique driven from Genetic Algorithms approach is called Linear Adaptive Genetic Algorithm (LAGA); pre-estimation optimization model that is based on Average Gradient Method (AGM) to accelerate the process of the optimization search engine; reliability model that is based on load resistance concept for calculating system reliability. Through this chapter the number of subjects will be discussed those are: background of water distribution systems, definition of problem in statement, main objectives, history of optimization and techniques, history of reliability and techniques, proposed optimization technique, the advantage of the new optimization technique, proposed reliability evaluation 
technique, proposed risk assessment technique, resultant decision support system, applications of the proposed model using existing cases studies, and conclusion including outcomes and recommendations. Developing the main model and it's sub-models in using MATLAB power with some snapshots will be discussed.

\section{Chapter objectives}

The objectives of this chapter have one common target that is to define the reliability-based optimization design for a water distribution network using modelling technique of MATLAB programming language. The following tasks have to be achieved:

1. Acquainting optimum least-cost design for water distribution networks using new efficient and time consumed method.

2. Define risk components for water distribution networks.

3. Define the most critical components of water distribution networks that affect the level of serviceability under different cases of operation (i.e. define level of service under risk).

4. Analysis, evaluation and treating reliability for water distribution networks.

5. Define the reliability of water distribution network over a given period of time.

6. Define the optimum solution of water distribution network that achieve the optimum lease-cost design and certain accepted reliability in one time (reliability-based optimization).

7. Develop stand alone reliability-based optimization model comprising all the above mentioned objectives

\section{Previous studies}

Solving the hydraulic equations for water distribution networks is a constrained non-linear hard programming problem (CNLHP) due to the nature of the non-linearity of the decision variables such as pipe diameters. For a given water distribution network, huge number of solutions could be selected through a range of the decision variables to select the best solution which arise the problem to be combinatorial optimization problems (Gupta and Kapoor 1994). Hamdy A. (1997) stated that some mathematical models may be so complex that is impossible to solve them by any of the available optimization algorithm and such cases heuristics are used instead of mathematical models to search for a good solution near the best or the optimum one. The advantage of heuristics over an exact optimization algorithm is that it is usually much faster to execute. Dorigo and Thomas (2004) stated that recently, many researchers have focused their attention on a new class of algorithms called meta-heuristics. A meta-heuristic is a set of algorithmic concepts that can be used to define heuristic methods applicable to a wide set of different problems. The use of meta-heuristics has significantly increased the ability of finding very high quality solutions to hard, practically relevant combinatorial optimization problems in reasonable time. Intelligent algorithms models are becoming essential tool to solve such non-linear hard problems for water distribution networks. Number of intelligent algorithms had been developed based on the meta-heuristic concept such as Simulating Annealing (SA), Tabu Search (TS), Guided Local Search (GLS), Greedy Randomized Adaptive Search Procedure (GRASP), Iterated Local Search (ILS), Evolutionary Computation (EC), Scatter Search, and Ant Colony Optimization (ACO). 


\section{Model components}

Getting the reliability-based optimization design for water distribution networks requires searching among a number of available population set of solutions, thus; RELOPT model consists of the following components (Moneim AM, 2009):

1. Hydraulic solver EPANET: consists of the dynamic libraries that are required to be called by MATLAB program for hydraulic analysis.

2. Pre-estimation model (AGM): this sub-model provides the lower and upper bounds that are required for OPTWNET to start optimization search process.

3. Optimization model (OPTWNET): defines the optimum solution using LAGA.

4. LAGA automatic search engine module.

5. Reliability model (RELWNET): this model is connected with three sub-models those are: minimum cut-sets model; Generic Expectation Function model; and reliability calculation model. The model passes the final calculated reliability to the main model RELOPT.

\subsection{Main model}

The main model is the main driver for RELOPT and contains all links to call or retrieve submodels or any external. Figure 1 represents the RELOPT model organization chart. The main model is coded in MATALB language and is designed to send and receive variables, input data, and outputs from different sub-models parties. The Main Model is playing a managing role between the different models by receiving and passing inputs and outputs between the models EPANET, AGM, OPTWNET, and RELWNET. Once a new water network had registered within EPANET environment, the input file name should be provided within the Main Model. During the run; EPANET is returning back an output file name to the Main Model. The Main Model is calling the pre-estimator AGM and receiving back the outputs which are the upper and lower bounds for LAGA search engine. The Main Model is passing the outputs from AGM to LAGA and receiving the outputs from LAGA which are the water network pipe diameters (decision variables). The Main Model is passing the water network data to the reliability model REWNET and receiving the calculated reliability measure of the current network in hand.

\subsection{Pre-estimation model}

The pre-estimation model is playing very important role in this application as it defines the upper and lower bounds for the optimization search process that is carried out by LAGA. Generally, defining the upper and lower bounds for optimization process decreases the search space which speeds up the optimization search and hence reduces the consumed run time. The pre-estimation model utilizes the Average Gradient Method (AGM) which depends on defining the critical node of minimum residual pressure within the network. The pre-estimator model AGM passes the upper and lower bounds to the optimization model OPTWNET to start searching of optimum solution among population of feasible solutions.

\subsection{Optimization model}

The optimization model OPTWNET uses LAGA and genetic optimization search engine. Figure 2 represents the OPTWNET model organization chart. The model RELWNET is running using the upper and lower bounds received from the pre-estimation model and passes out the pipe diameter decision variables to the main model. The number of generation 
used by LAGA is limited to 5 and the population size is limited to 10. Experiments have shown that these limits are enough for LAGA to define the global optimization solution for a water network. OPTWNET passes the resulting optimized decision variables to the main model which passes by its role to the reliability model RELWNET.

\subsection{Reliability model}

The reliability model RELWNET uses load-resistance principal to calculate the system reliability for water distribution networks (Moneim et al., 2010). Figure 3 represents RELWNET model organization chart. The RELWNET is dealing with the optimized decision variables obtained by OPTWNET. RELWNET is linked to sub-model Minimum Cut Set (MCS) to define the failure components. Following up the definition of failure components, Generic Expectation Function (GEF) sub-model is called by RELWNET to calculate the reliability of the network and passing the calculated reliability to the OPTWNET. OPTWNET is now assign penalty factor to each network passed on three constraints those are; pressure, velocity and reliability. The LAGA optimization search engine arranges the solutions by descending order through the model OPTWNET according to fittest solution. The optimum solution is passed back to the Main Model and final hydraulic check is carried out by EPANET.

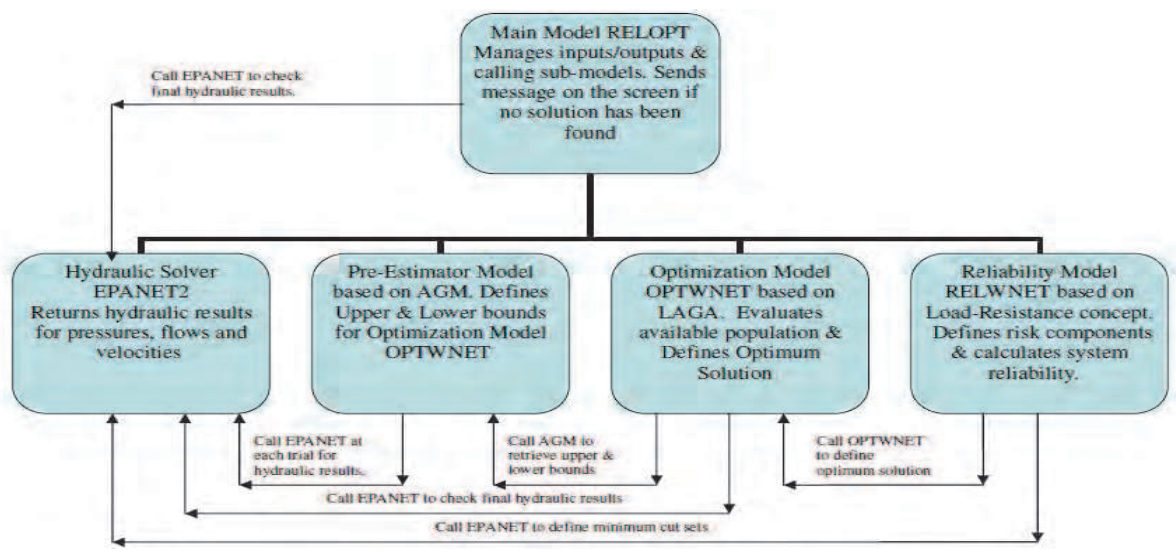

Fig. 1. Main Model Organization Chart (RELOPT).

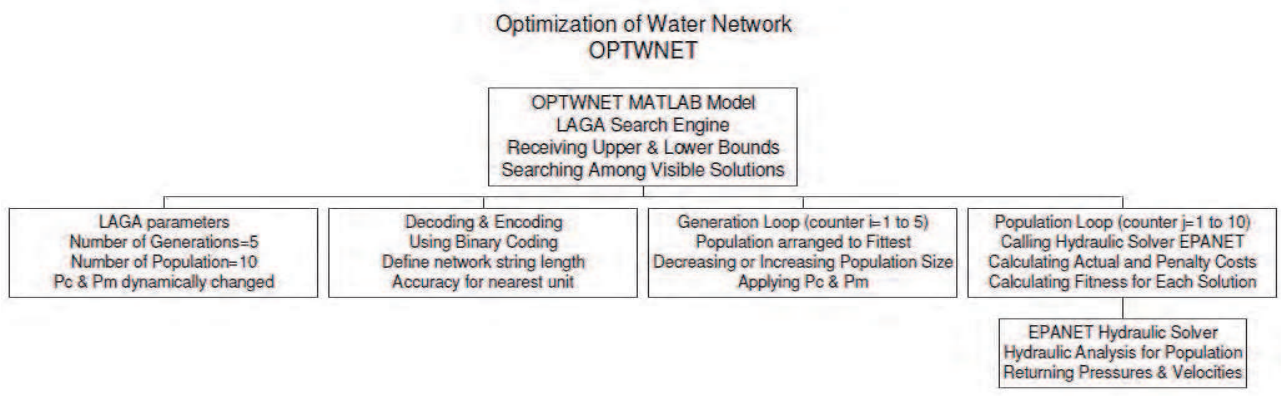

Fig. 2. OPTWNET Organization Chart. 


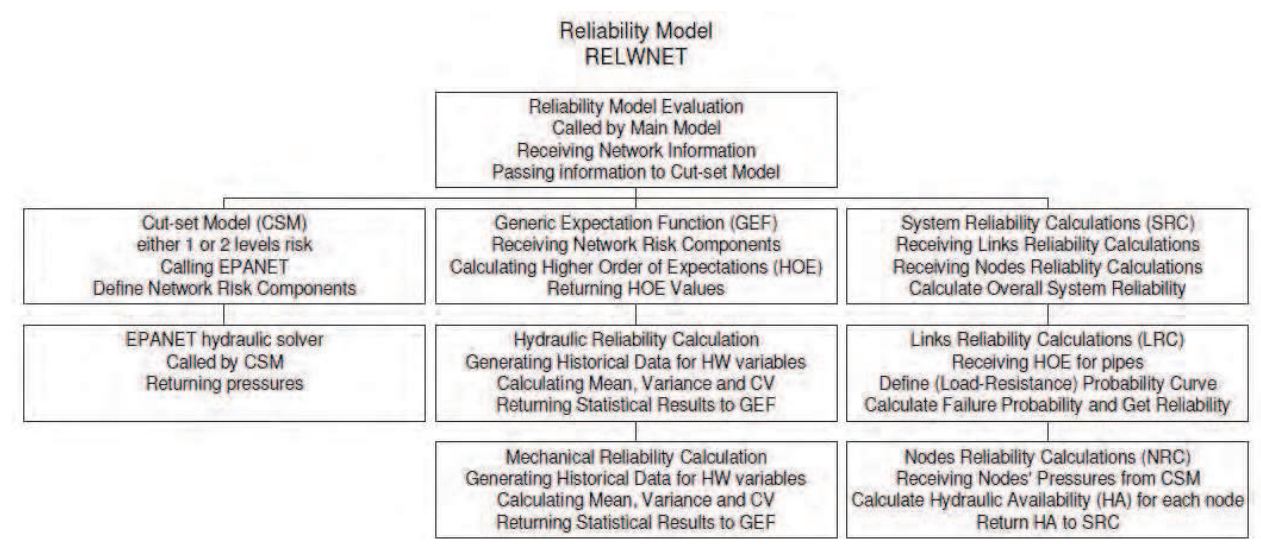

Fig. 3. RELWNET Organization Chart.

\section{Optimization technique}

Heuristics from Nature approach has been presented by (Colorni et al. 1992a, 1992b) presented methods that depend on as a non-derivative optimization method. The authors stated that Heuristics derived from Nature algorithms considered as a border between Operation Research (OR) and Artificial Intelligence (AI). These algorithms take inspiration from physics, biology, social, and use a certain amount of repeated trials for the NonProgramming Hard Combinatorial Optimization Problem (NPHCOP). Heuristics are obtained by one of the following application methods:

1. Using certain amount of repeated trials;

2. Employing one or more "agents" such as neurons, particles, chromosomes, ants, genetic algorithms, and so on;

3. Operating (in case of multiple agents) with a mechanism of competition-cooperation;

4. Embedding procedures of self-modifications of the heuristics parameters or of the problem representation.

\subsection{Genetic Algorithms (GA)}

Genetic Algorithms is one of the methods derived from Heuristics from Nature approach. GA is numerical optimization algorithms inspired by both natural selection and natural genetics. David A Coley (1999) demonstrated that GA is a general method capable of being applied to an extremely wide range of problems. David illustrates that GA has proved capabilities to solve many large complex problems where other methods experienced difficulties. Amongst many practical problems and areas which GA has been successfully applied are image processing; prediction of three dimensional protein structures; medicine; analysis of time series; solid-state physics; robotics; water distribution networks; training and designing artificial intelligence systems such as neural networks and control. David summarizes the main components of typical GA as follows:

1. A number or population of guesses of solutions to the problems;

2. A way or a method to evaluate how good or bad the individual solutions within the population;

3. A method for mixing fragments of the better solution to form new better solutions; 
4. A mutation operator to avoid permanent loss of diversity within the solutions.

David defined three main operators for the typical GA states below:

1. Selection: selection is used to apply pressure upon the population in a manner similar to that natural selection found in biological systems. Poorer performing individuals are disappearing out and the better is surviving. Better individuals are then having greater chance of performing new fitter genes;

2. Crossover: this operator allows solutions to exchange information in a way similar to that used by natural organism undergoing sexual reproduction. Information can be totally changed $(100 \%)$; fixed point crossover (constant percentage crossover) or variable point crossover (non-constant percentage crossover);

3. Mutation: mutation is used to randomly change (flip) the value of single bits within individual strings. The importance of mutation operator securing evolving of string that includes the global optimum as the mutation allows the population to "leapfrog" over the global optimum.

\subsection{Linear Adaptive Genetic Algorithm (LAGA)}

Attia and Horacek (2001) developed Linear Adaptive Genetic Algorithm (LAGA) to solve unconstrained optimization problems. The method is depending on applying modification to the internal Genetic Algorithm parameters like cross over and mutation probabilities based on generation index. The LAGA method is then becoming dynamically process and case sensitive with respect to the optimum solution.

The cross over probability rate $\mathrm{P}_{c}$ and mutation probability rate $\mathrm{P}_{\mathrm{m}}$ are concluded in GA operation to provide faster convergence when compared to constant probability rates. $P_{c}$ is set up to its highest value at the start of the optimization process and decreases linearly as the generation number is progressing.

$$
\mathrm{P}_{\mathrm{c}}=\frac{0.5}{\mathrm{M} 1}(\mathrm{~g} 1)+1
$$

Where: $g=$ number of the current generation,

$\mathrm{M}=$ total number of generation.

Unlike the crossover probability is the mutation probability which is not needed at the beginning of the optimization search as the population is very distinct. As the generation is progressing the solution starts to come slightly closer to the optimum, the mutation probability is then come to the view picture and starts from 0.005 and increased linearly up to 0.5 .

$$
\mathrm{P}_{\mathrm{m}}=\frac{0.005}{\mathrm{M} 1}(\mathrm{~g} 1)
$$

\subsection{Application of LAGA to water networks optimization}

LAGA approach has been employed for the first time in this research to solve the problem of water distribution network optimization. LAGA proved faster convergence and time consuming for optimization problems (Attia and Horacek, 2001) and it is prospective to solve water distribution network optimization problem. The first problem when applying LAGA for water distribution network optimization is that LAGA was adopted to solve direct unconstrained optimization problems. Hence, it is simple to define the population 
range and substitute directly in the objective function to evaluate the corresponding selected input variables. LAGA has been adopted to select the population for network solution (string of pipe diameters) and linked to hydraulic solver EPANET to evaluate each network solution according to predefined cost function. The second problem is that the search space for given pipe diameter limit was very large and that slowed down the convergence process. This problem had been eliminated by applying pre-estimation for the upper and lower bounds of diameter population as being explained in the forthcoming section 3.14. Figure 3.5 illustrates the flow chart for LAGA optimization technique. As being introduced in this chapter, the problem of water distribution networks need evaluation of each selected network solution by linking to hydraulic solver such as EPANET. This process is affecting the consumed run time for specific problem and as a result the expected run time to get the final solution will be much longer. The following advantages of LAGA technique are helping to reduce and accelerate the run time:

1. LAGA uses dynamic concept for the main parameters of GA process that allow changing the values of both cross over and mutation probability at the start of each generation. This mechanism is accelerating the searching process to allocate an optimum solution in shorter time than the traditional Genetic Algorithm;

2. LAGA reduces the population size as the optimization process tends to catch on the optimum solution. Conversely, LAGA increases the population size as the optimization process could not catch up an optimized solution. This mechanism adds another time consuming facility in case an optimum solution has been identified.

\section{Multiple optimization agents}

It has been noticed during the first run of LAGA for water network optimization that the search process for the optimum solution was not fast convergence and that because the difference between the upper and lower limits of the population search space was quite large. The lower and upper limits of the search space should conclude the available minimum and maximum commercial pipe diameter. This fact cause the search process to take time and the convergence is slow down. To eliminate unnecessary effort and time during the search process, pre-estimation optimization agent has been applied. The lower and upper bounds of the population search space can be defined. Moreover, it will be more efficient to apply the pre-estimation optimization process not only for the overall search space but also for each pipe within the network. The pre-estimation optimization process had accelerated the search process and reduced the required run time. New model had been developed to predict the lower and upper bounds of the search space and linked to LAGA main optimization model. That could be known as Meta-Model Development (MMD). The new model is called Average Gradient Method (AGM) for water networks optimization.

\subsection{Average gradient method for water networks optimization}

The average Gradient Method for water networks optimization is mainly depending on defining the most critical node within the water distribution networks and the critical path to the source node is then calculated. The method was driven from the principal that if the pressure at most critical node in the water network has been controlled to be greater than or equal to the minimum residual pressure, the resulting pipe diameter could be good estimation to start the optimization search process by LAGA. The following steps summarize the AGM: 
1. Define the required minimum pressure and let us refer it as (Pm);

2. Calculate the total required demands of the entire water network nodes and let us refer it as $(\mathrm{Qt})$;

3. Assuming flow velocity of $1 \mathrm{~m} / \mathrm{s}$, the corresponding pipe diameter (D) to convey the total required demand is then can be calculated;

4. Set all pipe diameters within the entire network to be equal to (D);

5. Calculate the corresponding pipe network cost and let us refer it as (Fo);

6. Solve the water network hydraulically using the hydraulic solver EPANET;

7. Define the most critical node within the entire network. The critical node is that node which has pressure less than the minimum residual pressure provided by the analyzer before the analysis starts;

8. Calculate the shortest path that lead from the defined critical node to the source node and let us refer it as (LS);

9. Calculate the head at the critical node $=\mathrm{Pm}+$ Node Elevation;

10. Calculate the difference between the source head and the critical node head $=$ Head at source - Head at critical node $=\mathrm{Hm}$;

11. Calculate the average gradient $(\mathrm{Gv})=\mathrm{Hm} / \mathrm{LS}$

12. Apply Hazen William formula for each pipe within the entire network using the calculated average gradient

$$
\mathrm{Q}=0.278 * \mathrm{C}^{*} \mathrm{D}^{2.63 *} \mathrm{G}_{\mathrm{v}}{ }^{0.54}
$$

Where $Q=$ resulting pipe flow for the current hydraulic analysis $(\mathrm{m} 3 / \mathrm{s})$

$\mathrm{C}=$ Hazen William friction factor

$\mathrm{D}=$ required pipe diameter to be calculated

$\mathrm{Gv}=$ average network gradient

1. The corresponding pipe diameter for each pipe within the network is then can be calculated;

2. Calculate the pipe network total cost (Fn);

3. Calculate the difference between (Fn) and (Fo). If the difference is greater than certain defined limit (say 1000 unit cost). Repeat steps from 6 up to 14. If the difference between (Fn) and (Fo) is less than or equal to the defined limit. Stop the process. The resulting pipe diameters are considered the lower bound for the search space population. The upper bound then is equal to 1.2 of the lower bound.

\subsection{Formulation of LAGA optimization technique}

Hydraulics of water distribution network is a complex task due to the non linear formulation of the pipe network flow functions. Consequently, applying optimization technique to search amongst available solutions to the assign the local or global optimum solution is not a simple or direct substitution task. GA search engine has the ability to pick up a certain number of available network solutions and throw the solutions into the population basket to evaluate each solution using the hydraulic solver EPANET and that is called the first generation for GA process. The evaluation process is based on how much the violation of each network solution about the pre-defined minimum pressure (desirable limits of serviceability). As long as the a certain solution is near to the desirable limits of serviceability the penalty factor is intended to be close to zero and vice versa, as long as the network solution is violated about the desirable limits of serviceability the penalty factor is 
intended to a value greater than zero. The objective function for water distribution networks is usually used as the cost function comprising the pipe lengths and diameters. The cost function can take the following general form:

$$
\operatorname{MIN}(F)=\sum_{\mathrm{i}=1}^{\mathrm{n}} \mathrm{C}_{\mathrm{i}}{ }^{*} \mathrm{D}_{\mathrm{i}}{ }^{*}{ }_{\mathrm{i}}+\mathrm{F}_{\mathrm{p}}+\mathrm{F}_{\mathrm{v}}
$$

Where $\mathrm{F}=$ total cost of the current network and MIN is referring to minimization.

$\mathrm{F}_{\mathrm{p}}=$ penalty cost due to pressure violation.

$\mathrm{F}_{\mathrm{v}}=$ penalty cost due to velocity violation

$\mathrm{C}=$ unit cost for each pipe diameter category

$\mathrm{D}=$ pipe diameter

$1=$ pipe length

$\mathrm{i}=$ pipe number

$\mathrm{n}=$ denotes to total pipe number within the network

\subsection{Adaptation of penalty cost for pressure violation}

The penalty cost can be applied according to the three cases those are:

Case of minimum allocated pressure $P_{\min }$ is greater than minimum desirable pressure $R_{p}$ and the difference does not exceed $10 \%$ :

The penalty cost $\mathrm{F}_{\mathrm{p}}$ is determined according to the following equation:

$$
\mathrm{F}_{\mathrm{p}}=\frac{\mathrm{F}}{\mathrm{R}_{\mathrm{p}}}\left(\mathrm{R}_{\mathrm{p}}-\mathrm{P}_{\min }\right)
$$

If the minimum allocated pressure of the current solution is greater than the desirable minimum pressure the resulting penalty cost will have negative value which will decrease the total corresponding cost function. Conversely, if the minim allocated pressure of the current solution is less than the desirable minimum pressure, the resulting penalty cost will have positive value which will increase the corresponding cost function.

1. Case of minimum allocated pressure $P_{\min }$ is less than minimum desirable pressure $R_{p}$ and the difference does not exceed $10 \%$ :

$$
\mathrm{F}_{\mathrm{p}}=\frac{\mathrm{F}}{\mathrm{R}_{\mathrm{p}}}\left(\mathrm{R}_{\mathrm{p}}-\mathrm{P}_{\min }\right) * 1000
$$

2. Case of more than $10 \%$ difference between the actual minimum pressure and the minimum desirable pressure and the solution is invisible:

$$
\mathrm{F}_{\mathrm{p}}=\frac{\mathrm{F}}{\mathrm{R}_{\mathrm{p}}}\left|\mathrm{R}_{\mathrm{p}}-\mathrm{P}_{\min }\right|{ }^{*} 1000
$$

3. Case number 2 and 3 will eliminate the solutions that violate about the optimum solution even if it is a feasible solution. The factor 1000 (this factor can be increased to any other value) will increase the cost value to an imaginary amount which secures disappearing of any violated solutions that exceed $10 \%$ of the desirable minimum pressure. 


\subsection{Adaptation of penalty cost for velocity violation}

The penalty cost can be applied according to the three cases those are:

1. Case of $10 \%$ difference between the actual maximum velocity and the maximum desirable velocity:

The penalty cost $\mathrm{F}_{\mathrm{v}}$ is determined according to the following equation:

$$
F_{v}=\frac{-1 * F}{V r}\left|V_{a}-V_{r}\right|
$$

Where $\mathrm{Vr}=$ desirable maximum velocity.

$\mathrm{V}_{\mathrm{a}}=$ Actual maximum velocity of the current solution.

If the actual maximum velocity of the current solution is greater or less than the desirable maximum velocity within a limit of $10 \%$ of the resulting penalty cost will have negative value which will decrease the total corresponding cost function.

2. Case of more than $10 \%$ difference between the actual maximum velocity and the maximum desirable velocity and the solution is visible:

$$
\mathrm{F}_{\mathrm{p}}=\frac{-1^{*} \mathrm{~F}}{\mathrm{Vr}}\left|\mathrm{V}_{\mathrm{r}}-\mathrm{V}_{\mathrm{a}}\right|{ }^{*} 1000
$$

Case number 2 will eliminate the solutions that violate about the optimum solution even if it is a visible solution. High velocities might cause pipe corrosion and high power loss. The factor (1000) will increase the cost value to an imaginary amount which securing disappearing of any violation solutions that exceed $10 \%$ of the desirable maximum velocity.

\section{Reliability calculation using load-resistance analysis}

The load-resistance analysis shall be applied to calculate the reliability of water distribution networks. It has been stated by (Y.K. Tung and Mays, 1985) that the resistance or strength of any component is defined as the ability of the component to accomplish its required mission with satisfactory state without a failure when subjected to an external stress. Stress is the loading of the component which may be a mechanical load, an environmental exposure, a flow rate, temperature fluctuation, etc. the stress loading tends to cause failure of the component. When the strength of the component is less than the stress imposed on it, the failure occurs. This type of analysis can be applied to the reliability analysis for components of water distribution systems. Equation 10 is a real translation to this concept stated very recently by (Yeou and Ben, 2005). The safety margin SM is a performance index that reflects the reliability performance of the network. Knowing the probability distribution for the (SM ) will help in computing the area under the probability curve that securing positive value of SM. The Safety Margin SM is solely the reliability of the system RS and can take the following form:

$$
\mathrm{RS}=\mathrm{P}(\mathrm{Y}>\mathrm{X})=\mathrm{P}(\mathrm{Y} \quad \mathrm{X}>0)
$$

Where $Y$ is the resistance of the system and $X$ is its loading. Accordingly, the reliability of a hydraulic system is defined as the probability of the resistance $Y$ to exceed the loading $X$. The resistance of a hydraulic system is essentially means the flow carrying capacity of the system and the loading is essentially the magnitude of the actual flows through the system that satisfy the required demands. Due to uncertainties of hydraulic behavior, the load and the resistance should be dealt as random variables and their probability distribution are 
essentially needed to develop a reliability model. The system Reliability RS can be calculated after the probability of system failure can be calculated. MATLAB built-in probability functions can be used to generate any number of historical data for both the load that is carried by a pipe and the resistance that is can be calculated using Hazen-William equation.

\subsection{Defining risk components using cut-set method}

It has been mentioned by (Moneim et al., 2010) that the cut-set method is powerful for defining the failure/risk components for complex systems such as water distribution networks. The cut set is defined as the set of system components which when failed causes the failure of the whole system. The cut set method utilize the minimum cut set that can be defined as the set which when failed cause the failure of the whole system but when any one component of the set has not failed, it does not cause failure of the system failure. The minimum cut set implies that all of its components must be in failure mode to cause failure of the whole system. Therefore component of a minimum cut set are effectively connected in parallel and each cutest is connected in series. The following form expresses calculation of reliability for a system:

$$
\mathrm{PF}=\sum_{\mathrm{k}=1}^{\mathrm{S}} \mathrm{P}\left(\mathrm{C}_{\mathrm{k}}\right)
$$

Where PF $=$ Probability of failure of the whole system

$\mathrm{P}\left(\mathrm{C}_{\mathrm{k}}\right)=$ Probability of failure for the minimum cut set number $\mathrm{k}$

$\mathrm{S}=$ total number of the minimum cut set.

It is easier to define the failure components and calculate the failure probability of each one, then the overall system failure cab be calculated simply according to the following sections.

\subsection{Combining hydraulic and mechanical reliability}

It has been stated by (Moneim et al., 2010) that hydraulic and mechanical failures are considered two independent events that might occur within water distribution networks. Hence, hydraulic and mechanical failures are two events statistically independent as the probability of any one of them is unaffected by the occurrence of the other. According to the multiplication law for independent events, the probability that two independent events will both occur is simply the product of their probabilities and that can be mathematically expressed according to the following equation:

$$
\mathrm{P}(\mathrm{A} \cap \mathrm{B})=\mathrm{P}(\mathrm{A}) \cdot \mathrm{P}(\mathrm{B})
$$

By defining the minimum cut-sets of a water distribution network, the overall failure probability for pipes and junctions within the water distribution network (PF) can be calculated. (Moneim et al., 2010) have developed the reliability model RELWENT which has been applied to calculate the reliability of water distribution networks. The overall system reliability can be expressed using the following equation:

$$
\mathrm{RS}=1-\mathrm{P}=1-\sum_{\mathrm{i}}^{\mathrm{M}}(1-\mathrm{HA}) * \mathrm{PF}
$$

Where: $\quad$ RS = Overall system reliability $\mathrm{P}=$ Overall system failure 
HA $=$ Hydraulic availability at junction nodes

$\mathrm{PF}=$ Combined hydraulic and mechanical failure probability for pipes.

In case the hydraulic availability at junction nodes has been found equal 1 (i.e $100 \%$ meets the required residual pressures), the term HA should be excluded from equation (13).

\section{Application example}

A simple 2-loop network represented in Figure 4 consists of 7 nodes, one reservoir and 8 pipes have been obtained from the literature (Alperovits \& Shamir, 1977). All pipes are equally length $1000 \mathrm{~m}$ and the Hazen-Williams coefficient of friction is assumed to be 130 for all pipes. The minimum required residual pressure for all demand nodes is $30 \mathrm{~m}$. The unit cost for pipe diameters is represented in Table 1 while Table 2 provides the data of nodes' demands and elevations.

\begin{tabular}{|c|c|c|c|c|c|c|c|c|c|c|c|c|c|c|}
\hline $\begin{array}{c}\text { Diameter } \\
\text { (In.) }\end{array}$ & 1 & 2 & 3 & 4 & 6 & 8 & 10 & 12 & 14 & 16 & 18 & 20 & 22 & 24 \\
\hline $\begin{array}{c}\text { Unit } \\
\text { Cost }\end{array}$ & 2 & 5 & 8 & 11 & 16 & 23 & 32 & 50 & 60 & 90 & 130 & 170 & 300 & 550 \\
\hline
\end{tabular}

Table 1. Unit Cost per meter length of pipe.

\begin{tabular}{|c|c|c|c|c|c|c|c|}
\hline $\begin{array}{c}\text { Node } \\
\text { Number }\end{array}$ & 1 & 2 & 3 & 4 & 5 & 6 & 7 \\
\hline $\begin{array}{c}\text { Demand } \\
(\mathrm{m} 3 / \mathrm{hr})\end{array}$ & -1120 & 100 & 100 & 120 & 270 & 330 & 200 \\
\hline $\begin{array}{c}\text { Elevation } \\
(\mathrm{m})\end{array}$ & 210 & 150 & 160 & 155 & 150 & 165 & 160 \\
\hline
\end{tabular}

Table 2. Node number, demands and elevation.

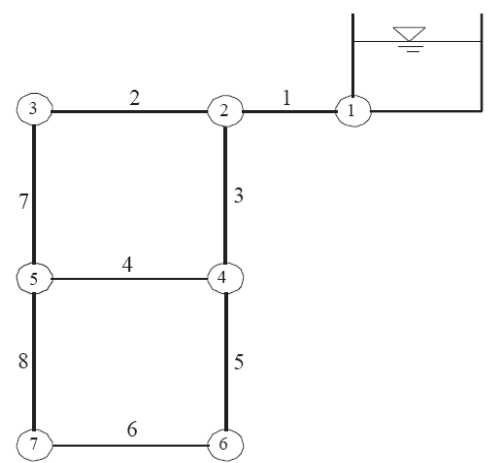

Fig. 4. Example 2-Loop Water Network.

\subsection{Solution steps}

The following steps are applied for the example application:

1. Open EPANET model interface, draw and create the input file format. 
2. Units are to be set to SI units.

3. Nodes should have consecutive numbers from 1 to the total number of nodes including the source node.

4. Pipes should have consecutive numbers from 1 to the total number of pipes.

5. Base demands and elevations should be given for all nodes.

6. Pipe lengths and friction factor should be given for all pipes.

7. No need to give any diameters for pipes at all.

8. Head for the source nodes should be given.

9. Create *.inp for the network and export by EPANET.

10. Open MATLAB environment and feed in the name of the *.inp input file in the main model.

11. An option input parameter for optimization and reliability in the same run needs to be fed in. Parameter of 1 is to be set if the reliability and optimization are required in the same run. 0 for optimization run only.

12. Save the main model and run.

\subsection{Solution criteria}

The model has been run to obtain the optimum design for the 2-loops example network Figure 1. The genetic parameters for LAGA search engine have the following criteria:

1. The number of generation for optimization $=2$

2. The number of population for optimization $=10$

3. The number of generation for reliability-based optimization $=2$

4. The number of population for reliability-based optimization $=6$

5. Limit of lower bound for decision variables (i.e. pipe diameter) $=90 \%$ of the resulted diameters obtained from the pre-estimator AGM model.

6. Limit of upper bound for decision variables (i.e. pipe diameter) $=110 \%$ of the resulted diameters obtained from the pre-estimator AGM model.

7. Minimum required system reliability $\mathrm{RS}=0.8$

8. Minimum required node reliability $\mathrm{RN}=0.85$

9. Minimum required residual pressure at all nodes $=30.0 \mathrm{~m}$

\subsection{Results interpretation}

The 2-loop water network example has been run for both optimization and reliability and the results are discussed in the following sections.

\subsubsection{Optimization results using 2 generations}

The results for optimization have obtained after 13.60 seconds using LAPTOP of $1.7 \mathrm{MHZ}$, and 2 G RAM. The cost of the optimum design is found 388,500 using the unit prices interpolation driven from Table 1 . The cost of the network according to the restricted list in Table 1 is found 447,000 with a minimum residual pressure $(29.6 \mathrm{~m})$. Although the obtained residual pressure is considered invisible, the run illustrates how much effective for the optimization model OPTWNET to consume run time. The search engine LAGA has improved the pre-estimated optimum cost by AGM model from 492,000 to 447,000. The

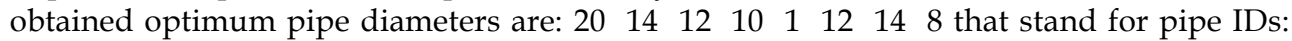
$\begin{array}{llllllll}1 & 2 & 7 & 8 & 6 & 5 & 3 & 4\end{array}$. Hydraulic results of this optimization process are illustrated in Appendix A. 


\subsubsection{Optimization results using minimum 100 generations}

The results for optimization have obtained after 135.34 seconds ( 2.25 minutes) using of the same previous configuration. The cost of the optimum design is found 427,000 using the unite cost of the commercial diameters illustrated in Table 1. The minimum obtained residual pressure is $(30.13 \mathrm{~m})$. The optimum pipe diameters for this run are: $18,14,14,10,1$, 14,14 , and 8 those stand for the pipe IDs: $1,2,7,8,6,5,3$ and 4 .

\subsubsection{Reliability results}

The results for reliability-based optimization have obtained after 34.3 seconds using LAPTOP of the same configuration stated above. The cost of the optimum solution based reliability is found 1,288,000 (i.e. 300\% increased comparing to optimization-based cost only) with system reliability 0.951 and nodal reliability 0.9668 while the original system and nodal reliability are 0.8708 and 0.93 respectively. The results for the obtained reliability versus residual pressure and cost are illustrated respectively through Fig. 5 and Fig. 6.

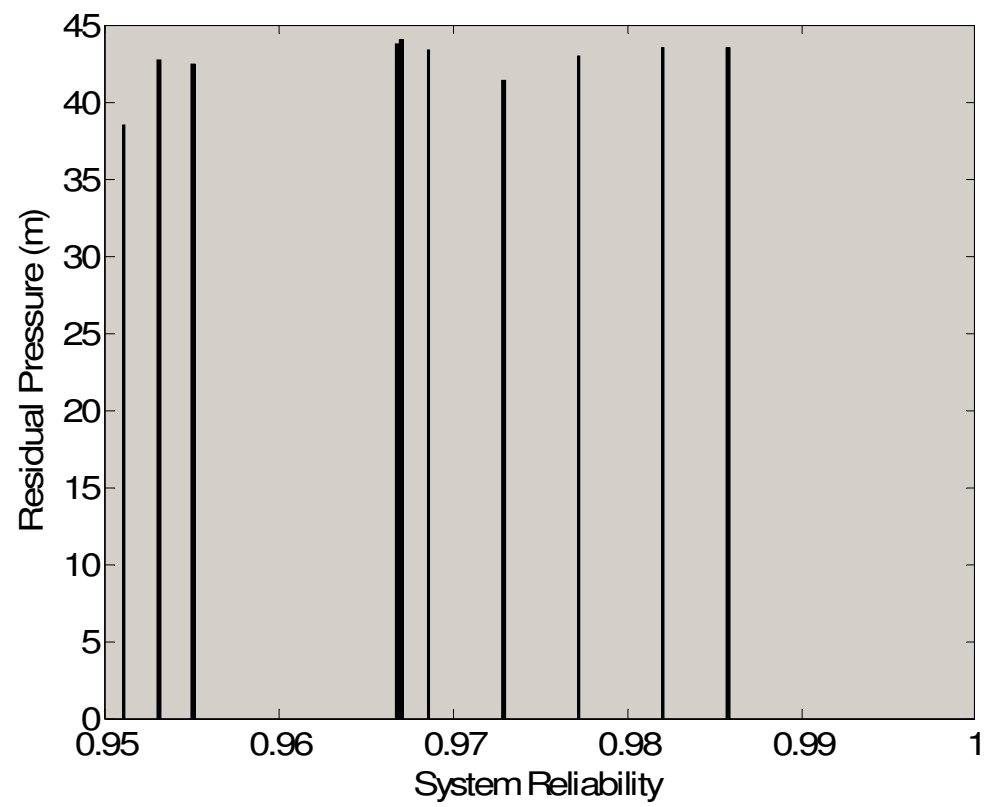

Fig. 5. Reliability versus Residual Pressure for 2-loop Network.

\subsubsection{Getting reliability for the optimum solution}

The water network of 2-loops has been run to get the reliability of the optimum design obtained in previous section. The reliability of the obtained optimum solution has found 0.8708 and the nodal reliability for the network nodes are $\begin{array}{lllll}0.9912 & 0.9609 & 0.9456 & 0.8708\end{array}$ 0.9609 0.9039. The condition of getting optimum design-based cost doesn't mean that network is reliable. According to this fact, specific reliability level should be defined before applying reliability-based optimization model for a water distribution network. 


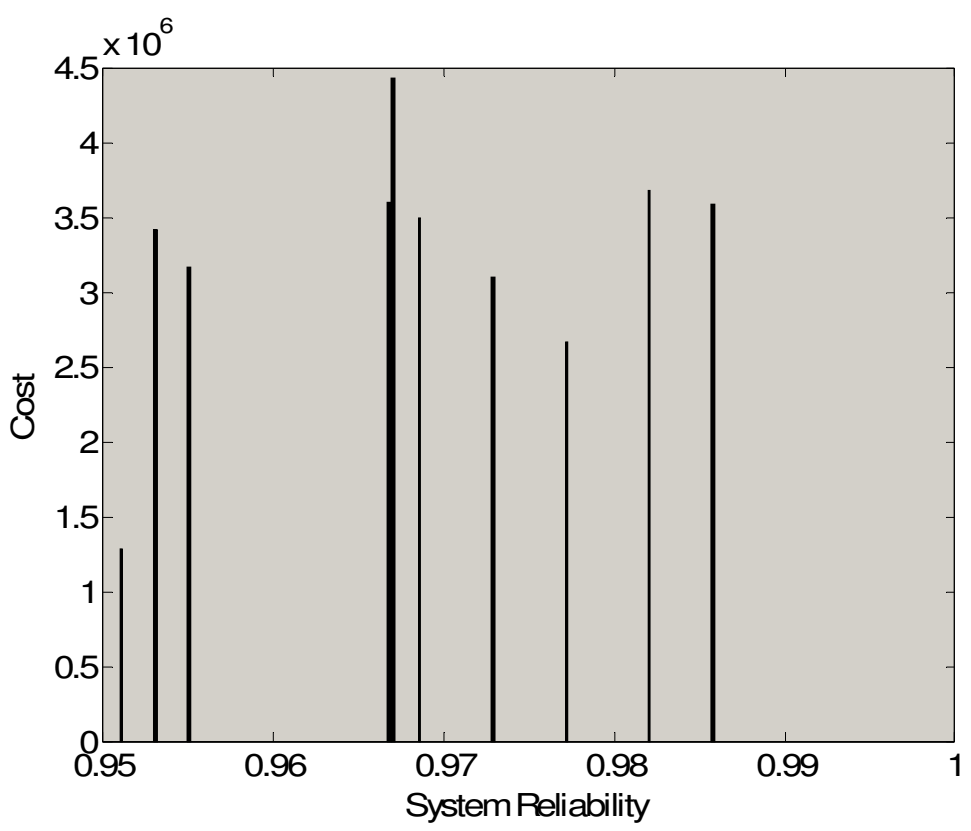

Fig. 6. Reliability versus Cost for 2-Loop Water Network.

\subsubsection{Characteristic reliability-cost relationship}

To verify the relationship between the reliability and the cost of the water distribution network, several runs have implemented under different reliability constraints starting from 0.5 up to 0.95 . The following cost equation has been obtained by applying curve fitting to the data of Table 1:

$$
\mathrm{C}=\sum_{\mathrm{i}=1}^{\mathrm{N}} 6.85^{*} \mathrm{e}^{0.1312 \mathrm{D} * \mathrm{~L}}
$$

Where: $D_{i}=$ Pipe diameter in inches for pipe and $L_{i}$ is the pipe length in meters.

The results indicated that relationship between the cost and the reliability is directly proportional which is logically expected. The point of issue is that a characteristic equation can express the relationship between the cost and reliability and accordingly the cost can be calculated for a given value of reliability. This can help when specific allocated budget needs to be adopted for a given water network. Fig. 7 illustrates the relationship between the cost and system reliability for the 2 loop water network. 


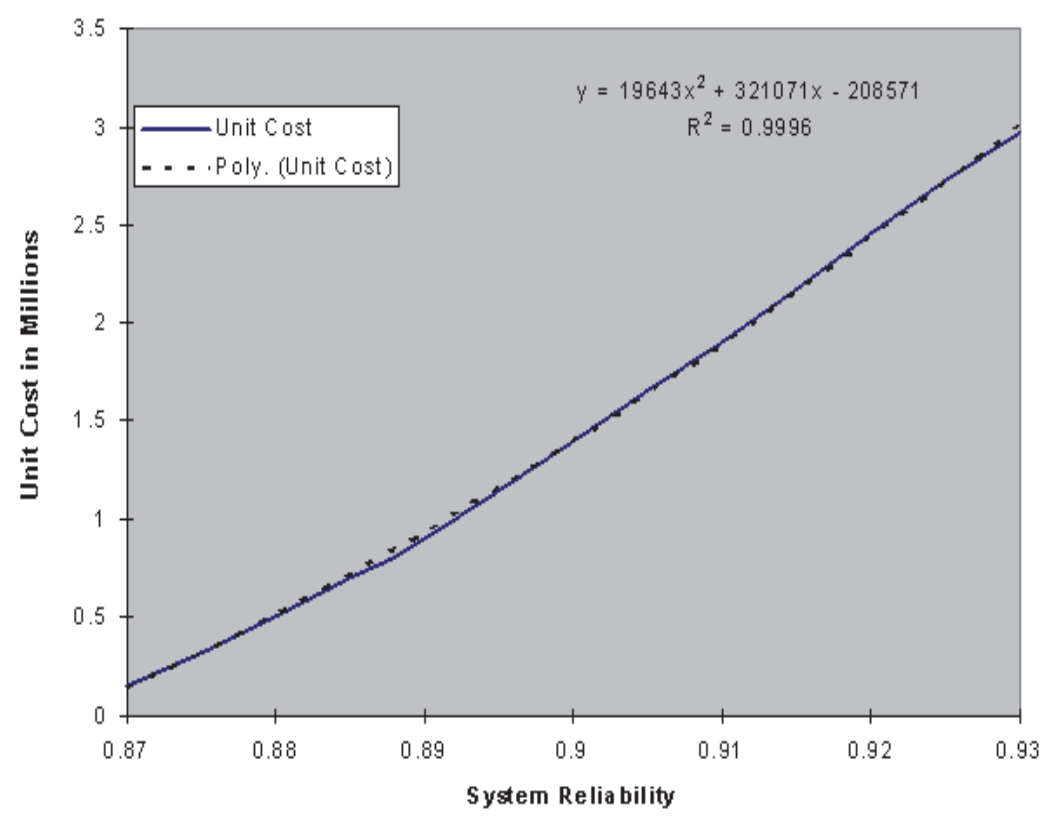

Fig. 7. Characteristic Reliability-Cost for 2-Loop Water Network.

\section{Conclusion}

In this chapter new model for reliability-based optimization has been developed using MATLAB programming language to define an optimum and reliable design for water distribution networks. The results indicated that the model RELOPT provides acceptable level of confidence when has applied to reliability-based optimization problems. RELOPT consist of four models those are: Pre-estimation mode called Average Gradient Model (AGM); hydraulic solver EPANET; optimization model OPTWNET based on new genetic algorithm concept called Linear Adaptive Genetic Algorithm (LAGA); and Reliability model RELWNET based on load resistance concept for reliability evaluation. The minimum cut sets method has been adopted to define the risk components for complex systems such as water distribution networks. Generic Expectation Functions (GEF) has been adopted to define the probability distribution for the difference between the load and resistance and hence the failure probability has been calculated to calculate the system reliability. It has been illustrated through that achieving reliability-based optimization represents the optimum solution for water distribution networks when considered as constraint during the optimization search process. Optimization of design-based cost is normally securing the function of the water distribution networks during normal operation while reliability-based optimization is normally securing the function of the water distribution network under risk. Giving that the risk has been occurred is an important approach to apply during the design of water distribution networks especially for large scale networks. Optimization cost for water networks could save nations' budget while designing a new network but shall not securing strategic decisions under certain risks or failures. On another point of view, while 
certain failure has occurred, budget may exceed normal levels to overcome such risks. Reliability-based optimization helps decision makers to adopt pre optimum strategic maintenance/operation programs or emergency scenarios to overcome lots of problems caused by network failures. Increasing the current construction budget for optimization based cost design of a water network to accommodate its reliability, will save more investment in future giving that certain risks have occurred.

\section{Acknowledgment}

The author is so grateful to his professors Dr. Ahmed Moawad who has supported me along my researches development with his value time and advices. The author also submit his greetings to his professors Dr. Anas Al Molla, Dr. Ayman Al Salawy, Dr. Abdel Badee Salem and Dr. Amgad El-Ansary who have given their value advices and encouraging through my research and life of experience. Great thanks to everybody who has helped me with an advice, time, word and/or technical data which with no doubt has a positive effect to improve my work.

\section{References}

Alperovits E. and Shamir, U., (1977). Design of optimal water distribution systems. Water Resources Research, Vol. 13(6), pp. 885-900.

Attia A. and Horacek P., (2001). Adaptation of genetic algorithms for optimization problem solving. 7th Intern. Mendel conference on soft computing pp. 36-41, Brno, Cizek Republic.

Colorni, A., M. Dorigo and V. Manniezzo (1992a). Distributed optimization by ant colonies. In: Proceedings of the First European Conference on Artificial Life (ECAL-91) (F.J. Varela and P. Bourgine, Ed.). The MIT Press. Cambridge MA. pp. 134142.

Colorni, A., M. Dorigo and V. Manniezzo (1992b). An investigation of some properties of an ant algorithm". In: Parallel problem solving from nature, Vol 2. (R. M"anner and B. Manderick, Ed.). North-Holland. Amsterdam, pp. 509-520.

David A Coley, 1999. An Introduction to Genetic Algorithms for Scientists and Engineers. World Scientific Publishing Company.

Dorigo, M., and Thomas, S., (2004). Ant colony optimization. MIT Press, Cambridge, Massachusetts, London, England.

Gupta, I., Gupta, A., and Khanna, P. (1999). Genetic algorithm for optimization of water distribution systems. Environmental Modelling \& software Vol.-4, pp. 437-446.

Hamdy, A. T., 1997. Operation research an introduction. Sixth Edition, Prentice-Hall International, Upper Saddle River, New Jersey.

Moneim A.M., Moawad A.K., Molla A., and Selawy A. (2010). RELWANET: Reliability Evaluation Model for Water Distribution Networks. Australian Journal of Water Resources, Volume 14 No. 1.

Su, Y. C., Mays, L. W., Duan, N. and Lansey, K. E. (1987). Reliability-based optimizationmodel for water distribution system. Journal of Hydraulic Engineering, ASCE, 114(12), 1539-1556. 
Y. K. Tung, (1985). Evaluation of water distribution network reliability. Hydraulics and Hydrology in the Small Computer Age, Proceedings of the Specialty Conference, American Society of Civil Engineers, Hydraulics Division, Vol.1, Lake Buena Vista, Florida, August 12-17, 1985. 


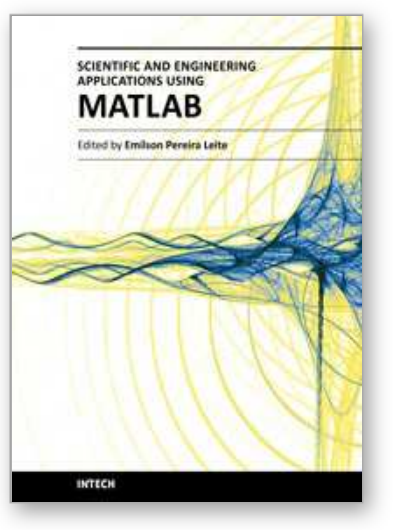

\author{
Scientific and Engineering Applications Using MATLAB \\ Edited by Prof. Emilson Pereira Leite
}

ISBN 978-953-307-659-1

Hard cover, 204 pages

Publisher InTech

Published online 01, August, 2011

Published in print edition August, 2011

The purpose of this book is to present 10 scientific and engineering works whose numerical and graphical analysis were all constructed using the power of MATLABÂ® tools. The first five chapters of this book show applications in seismology, meteorology and natural environment. Chapters 6 and 7 focus on modeling and simulation of Water Distribution Networks. Simulation was also applied to study wide area protection for interconnected power grids (Chapter 8) and performance of conical antennas (Chapter 9). The last chapter deals with depth positioning of underwater robot vehicles. Therefore, this book is a collection of interesting examples of where this computational package can be applied.

\title{
How to reference
}

In order to correctly reference this scholarly work, feel free to copy and paste the following:

Mohamed Abdel Moneim (2011). Modelling Reliability Based Optimization Design for Water Distribution Networks, Scientific and Engineering Applications Using MATLAB, Prof. Emilson Pereira Leite (Ed.), ISBN: 978953-307-659-1, InTech, Available from: http://www.intechopen.com/books/scientific-and-engineeringapplications-using-matlab/modelling-reliability-based-optimization-design-for-water-distribution-networks

\section{INTECH}

open science | open minds

\section{InTech Europe}

University Campus STeP Ri

Slavka Krautzeka 83/A

51000 Rijeka, Croatia

Phone: +385 (51) 770447

Fax: +385 (51) 686166

www.intechopen.com

\section{InTech China}

Unit 405, Office Block, Hotel Equatorial Shanghai

No.65, Yan An Road (West), Shanghai, 200040, China

中国上海市延安西路65号上海国际贵都大饭店办公楼 405 单元

Phone: +86-21-62489820

Fax: $+86-21-62489821$ 
(C) 2011 The Author(s). Licensee IntechOpen. This chapter is distributed under the terms of the Creative Commons Attribution-NonCommercialShareAlike-3.0 License, which permits use, distribution and reproduction for non-commercial purposes, provided the original is properly cited and derivative works building on this content are distributed under the same license. 